\title{
Early metal enrichment in high-redshift quasars
}

\author{
R. Maiolino \\ Astronomical Observatory of Rome, via di Frascati 33, 00040 Monte Porzio Catone, Italy
}

\begin{abstract}
Quasars are powerful systems whose spectrum is rich of metal features that allow us to investigate the chemical evolution of galaxies at very high redshift, even close to the reionization epoch. I review the main observational constraints on the metallicity of quasars host galaxies at high redshift and discuss the implications and issues for models of galaxy evolution in the early universe.
\end{abstract}

Keywords: galaxies: abundances, galaxies: high-redshift, quasars: emission lines

PACS: 98.54.Aj - 98.58.Ay - 98.62.Bj- 98.62.Js

\section{INTRODUCTION}

Thanks to their huge luminosity, quasars deliver high signal-to-noise spectra even at very high redshift. The most distant quasars currently known are at $\mathrm{z} \sim 6.4$, at the end of the re-ionization epoch [1,2]. The several metal features observed in their spectrum provide precious information on the metal enrichment of their host galaxies, which is linked to their past star formation history. Luminous quasars and radio galaxies are generally hosted in very massive systems, therefore they offer the possibility to investigate the metallicity evolution of the most massive representatives of the galaxy population at any epoch. Generally, metallicity information on quasars is inferred from the analysis of the broad emission lines, which are very strong, but are emitted from a very small nuclear region. Metallicity information on the scales of the host galaxy can be inferred from the narrow emission lines, which are emitted from a region ranging from a few $100 \mathrm{pc}$ to several kpc in size; however narrow lines at high-z are generally detectable only in those systems where the broad lines are obscured, i.e. type 2 quasars and narrow line radio galaxies. An alternative method to investigate the metallicity of quasars host galaxies is to measure metallicity tracers emitted from the host galaxy, not excited by the nuclear accreting black hole, but powered by star formation; however these tracers are generally observable in the millimeter and far-IR spectral ranges, where observations are still limited to a few, extremely bright objects. Each of these metallicity measurements provides precious and complementary information on the evolutionary stage and star formation history of quasar host galaxies in the early universe. In this paper I review these various observational results on the metallicity enrichment of quasars and radio galaxies, focusing on the most distant systems known, and I review some important implications of these observational findings for our understanding of galaxy formation in the early universe. 


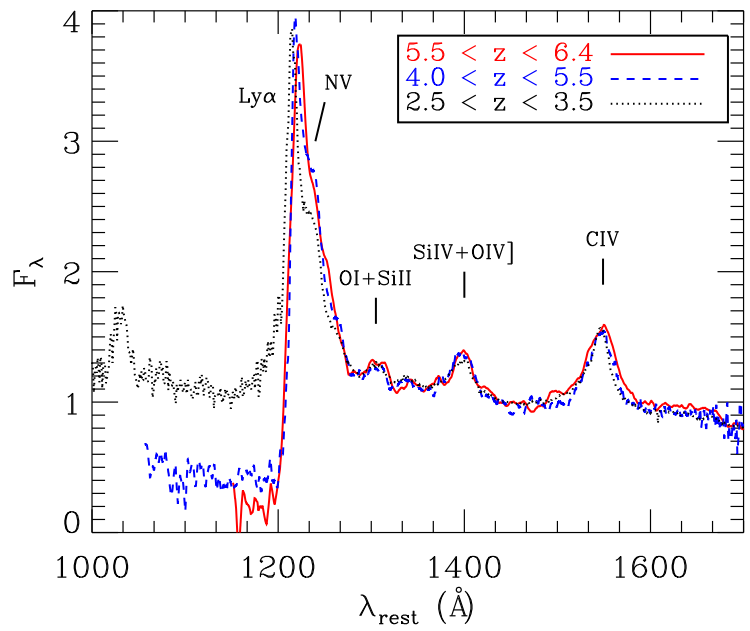

FIGURE 1. Stacked spectra of quasars in different redshift bins (from Juarez et al. [9]). Note that the relative intensity of the metal lines (and in particular the (SiIV+OIV])/CIV ratio) remains constant over the wide redshift interval $2.5<\mathrm{z}<6.4$, indicating that the metallicity in the observed quasars does not evolve with redshift.

\section{OBSERVATIONAL CONSTRAINTS ON THE METALLICITY OF HIGH-Z AGN}

\section{The metallicity of the BLR in high-z quasars}

Thanks to the large spectroscopic SDSS quasar sample, and by exploiting complex photoionization models, it has been possible to constrain the metallicity of the Broad Line Region (BLR, i.e. the innermost ionized region) in large samples of quasars in the redshift range $2<\mathrm{z}<4.5$ (which is the redshift range where the main UV broad lines, used to constrain the BLR metallicity, are observable in the optical) [3, 4]. In most cases the BLR metallicity is inferred to be several times solar. Moreover, there is a clear dependence of the BLR metallicity on the quasar luminosity. The metallicityluminosity relation may actually result from a black hole-metallicity dependence [5] or from a relation between accretion rate $\left(\mathrm{L} / \mathrm{L}_{E d d}\right)$ and metallicity [6]. However, an interesting result is that, once the metallicity-luminosity relation is accounted for, there is no evidence for any evolution of the BLR metallicity with redshift [4]. At redshift $\mathrm{z}>5$, i.e. approaching the re-ionization epoch, determining the metallicity of the BLR is more difficult, since several of the emission lines used to constrain the metallicity are shifted into the near-IR. Early near-IR spectroscopic studies found evidence for very high metallicities of the BLR in a few z $\sim 6$ quasars [7, 8]. More recently, Juarez et al. [9] used near-IR and optical spectra to constrain the metallicity of 30 quasars in the redshift range $4<\mathrm{z}<6.4$. They confirm very high metallicities and no evidence for any metallicity evolution when compared with lower redshift quasars (Fig.1).

The broad emission lines can also provide clues on relative chemical abundances. In particular, the abundance of iron and carbon, which are subject to a delayed enrichment relative to $\alpha$-elements, provides important information on the star formation history 
in quasar hosts. The UV spectrum of quasars is characterized by a prominent $\mathrm{Fe}$ bump, around 2300-3100 A, due to the blend of several thousand FeII transitions, whose ratio with the $\mathrm{MgII} \lambda 2798$ doublet is used to trace the Fe/ $\alpha$ abundance ratio. However, inferring an absolute value of $\mathrm{Fe} / \alpha$ from the FeII/MgII intensity ratio is very difficult, since the latter depends also on the physical conditions of the BLR [10]. As a consequence, the FeII/MgII is often take as a tracer of the relative variations of $\mathrm{Fe} / \alpha$. Several studies have been published reporting measurements of FeII/MgII in high redshift quasars $(3<\mathrm{z}<6.4)$, by using deep near-IR spectroscopic observations $[11,12,13,14,15,16]$. All of these studies consistently find that there is no evolution of the FeII/MgII ratio as a function of redshift, out to $\mathrm{z}=6.4$. A similar result is obtained for the carbon abundance: the intensity of CIV $\lambda 1549$ and CIII] $\lambda 1900$ relative to other lines suggest that the abundance of carbon relative to other elements remains constant out to $\mathrm{z}=6.4$. I will discuss the implications of these findings in the next section.

\section{Metallicity in the NLR of high-z AGNs}

The BLR is a very small region which may not be representative of the metallicity in the host galaxy. Gas on large scales, in the host galaxy, ionized by the AGN produces the so called Narrow Line Region (NLR). These narrow lines can generally be measured more easily if the nuclear region is obscured, i.e. in type 2 AGNs. However, in high redshift objects (mostly radio-galaxies and type 2 QSOs) there are often only a few, faint narrow lines detected that can be used to constrain the NLR metallicity [17, 18, 19, 20]. As a consequence of the faintness of the narrow lines, the sample of high-z objects for which the NLR metallicity has been investigated is much smaller ( 60 objects) than the broad line sample, and currently limited to $\mathrm{z}<4$. By exploiting photoionization models to interpret the narrow line ratios, it is found that the metallicity of the NLR is generally lower than typically observed in the BLR, but still super-solar. On average there is no evidence for any evolution with redshift of the NLR metallicity. It is also interesting to note that the lack of evolution observed in the NLR applies also to the CIV $\lambda 1549 / \mathrm{HeII} \lambda 1640$ narrow line ratio, suggesting a lack of evolution of the carbon abundance, as for the BLR, but on much larger scales.

\section{Abundances from the associated absorption systems}

The resonant (UV) narrow absorption lines due to the interstellar medium of quasars host galaxies provide a powerful tool to investigate the chemical abundances. When detected, these absorption systems can in principle provide very accurate measurements of the abundances of individual elements, since we can directly measure the column of each element (provided that the absorption lines are not heavily saturated and that the ionization corrections can be inferred). In practice, this approach requires high spectral resolution and high $\mathrm{S} / \mathrm{N}$ spectra, resulting in a small number of quasars where this strategy can be pursued, most of which at $\mathrm{z}<3$. Generally quasars associated absorption systems show super-solar metallicities, even for what concerns the carbon abundance 
(see [21, 22] and references therein), supporting the findings obtained for the NLR with higher precision. However, it is important to bear in mind two caveats. The location of the absorber is unknown; we only know that it cannot be too close to the nuclear source based on the line width and also based on the ionization structure (in some cases inferred lower limits on the distance from the nuclear source are $>100 \mathrm{kpc}$ ). As a consequence we do not really know whether the gas being probed in absorption is part of the quasar host galaxy, or gas clouds in their halo or in the galaxy outskirts. For what concerns the carbon abundance, it is important to bear in mind that dust depletion reduces the column of carbon measured from the absorption systems. Hence, the carbon abundance inferred with this method is actually a lower limit.

\section{Metallicity constraints from (sub-)mm observations}

Recent $\mathrm{mm}$ and submm observations have delivered precious information on the interstellar medium of high-z quasar host galaxies. The detection of strong $\mathrm{CO}$ emission in some quasars at $\mathrm{z}>5[23,24,25]$, as well as the detection of the [CII] $158 \mu \mathrm{m}$ line [26, 27, 28], with intensities comparable to lower redshift and local galaxies (with similar star forming rates), strongly suggests that the host galaxy of these distant quasars is highly metal enriched, and especially in terms of carbon abundance. These data are still far from providing accurate measurements of the metallicity and of the chemical abundances, however future facilities (e.g. ALMA, SPICA) will allow us to measure additional transitions (such as $[\mathrm{OI}] 63 \mu \mathrm{m},[\mathrm{NII}] 122 \mu \mathrm{m}$ and other molecular transitions) that will provide constraints the metal abundances in the ISM [29].

Another indication of high metal abundances in distant quasars is the detection of huge of dust mass masses (exceeding $10^{8} \mathrm{M}_{\odot}$ ), as inferred from the mm-submm continuum (far-IR rest-frame) even in the most distant quasars known, close to the reionization epoch [30, 31]. Inferring the metallicity from the dust content is difficult at high redshift for various reasons: the dust composition is not well known [32, 33, 2]; it is not clear what fraction of the metals have been locked in dust; finally, both dust mass and gas mass (when available) are subject to large uncertainties. However, rough estimates suggest that the observed dust masses must be associated to an ISM with solar or supersolar metallicity. If confirmed with more accurate measurements, these findings provide further evidence that even the host galaxies of quasars close to re-ionization $(\mathrm{z} \sim 6)$ have reached high metallicities in these early epochs in the universe, confirming the results obtained for the BLR, but on much larger scales.

\section{INTERPRETATION AND IMPLICATIONS FOR GALAXY EVOLUTION}

\section{The apparent lack of metallicity evolution}

The apparent lack of redshift evolution of the BLR metallicity may be deceiving. It does not imply that the metallicity of the BLR in individual AGNs does not evolve with 
time. It simply means that quasars that are detectable (with a given luminosity) at any epoch have reached the same metallicity regardless of their redshift. This effect probably results from a combination of selection effects and the evolutionary link between supermassive black hole and their host galaxies. Indeed, in a flux-limited survey, quasars can only be detected if they reach a minimum luminosity, which translates into a minimum black hole mass (even if they are accreting at the Eddington rate). Most models predict that the quasar host galaxy evolve along with the nuclear black hole [34, 35, 36, 37, 38], so to match the local $\mathrm{M}_{\mathrm{BH}}-\mathrm{M}_{\mathrm{gal}}$ relation. As a consequence, by the time a quasar has reached the luminosity to be detected, its host galaxy has already evolved and enriched the interstellar medium. To illustrate this effect more clearly, Fig. 2 (from [9]) shows the evolution of the metallicity, of the quasar luminosity and of the gas mass according to one of the theoretical models [34]. The shaded area indicates the epoch when the high-z quasar is luminous enough to be detected in the SDSS survey; by the time the quasar has reached this limit the host galaxy has already reached a metallicity of three times solar. Obscuration may be another parameter introducing additional selection effects. Models expect that during the early phases the quasar is heavily embedded in gas and dust, making it unobservable at optical wavelengths. Only during the latest stages the quasar wind is powerful enough to expel large quantities of dust and gas, making the quasar detectable by optical surveys. The hatched region in Fig. 2 shows the epoch when the quasar is unobscured (when more than half of the gas mass has been expelled), corresponding to an epoch when the gas metallicity has reached $\sim 4 \mathrm{Z}_{\odot}$.

The finding that the BLR metallicities of quasars observed at different redshifts have on average the same metallicity implies that the evolutionary BH-galaxy connection occurs in a similar manner at any epoch, regardless of redshift. In particular, the finding that even in $\mathrm{z} \sim 6$ quasars the metallicity is similar to lower redshift quasars implies that the same BH-galaxy co-evolutionary mechanism is already at work right after reionization. The very high metallicity observed at $\mathrm{z} \sim 6$ indicates that the host galaxies of these quasars are already highly evolved (at least from the chemical point of view) soon after re-ionization. These objects probably represent the most extreme cases of galaxy downsizing (at least in its chemical version [39]), i.e. these are the most extreme examples of massive galaxies forming very rapidly at very high redshift.

Note that the same reasoning can explain the apparent lack of metallicity evolution for the NLR of radio-galaxies and obscured quasars. Obviously, for these classes of AGNs the selection effects due to obscuration are unlikely to play a significant role. However, the requirement of a minimum power to be detected in radio or X-ray surveys (hence minimum black hole mass) probably produces the same selection effect in terms of NLR metallicity as for the BLR.

\section{The extreme metallicities in the BLR}

According to the photoionization models, the metallicity in quasar nuclei, as inferred from the broad lines, is extremely high: $\mathrm{Z}_{\mathrm{BLR}} \sim 7 \mathrm{Z}_{\odot}$ for the most distant quasars [9] and in some cases approaching $\mathrm{Z}_{\mathrm{BLR}} \sim 10 \mathrm{Z}_{\odot}$. These metallicities are not unrealistic. Indeed, the BLR is a very small region, containing only $\sim 10^{4} \mathrm{M}_{\odot}$ of gas; therefore, even a few 


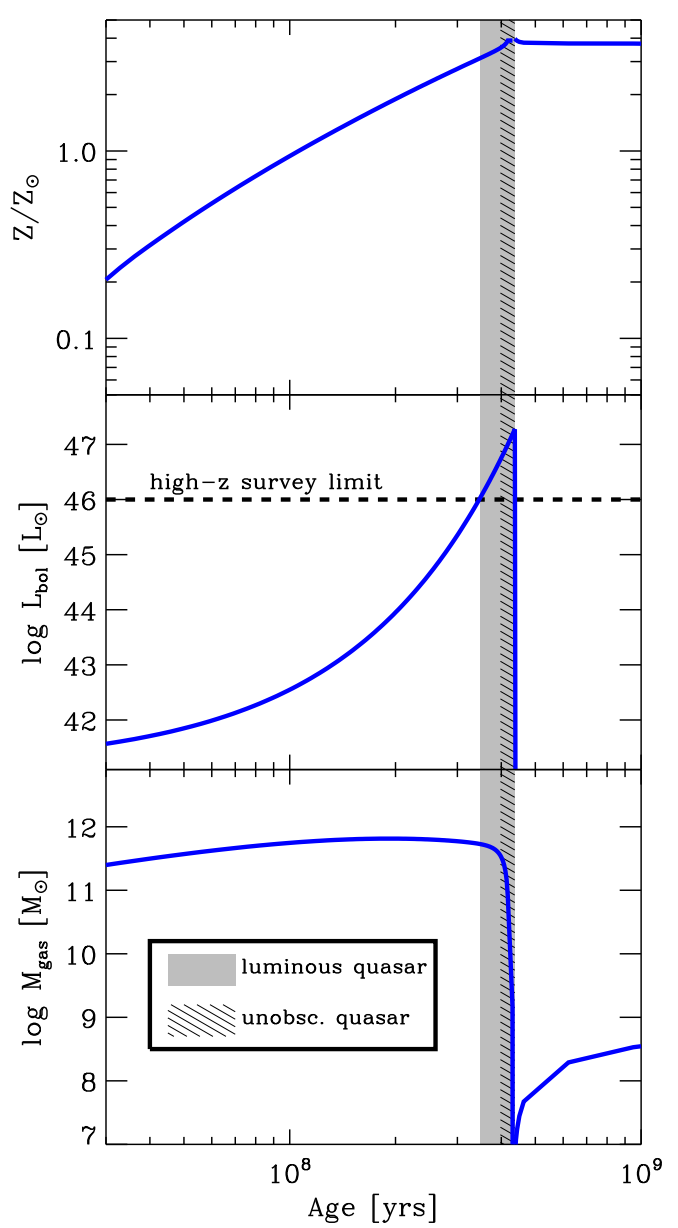

FIGURE 2. Evolution of the gas metallicity, bolometric quasar luminosity and gas mass according to the model in [34]. The horizontal dashed line shows the approximate minimum bolometric luminosity detectable at high-z in the SDSS quasar survey. The shaded area shows the epoch when a high-z quasar is luminous enough to be detected in the SDSS survey (if unobscured). The hatched area is the epoch when the quasar is likely unobscured and therefore detectable in optical surveys. By the time the quasar has reached the luminous, "unobscured" epoch the ISM is already highly enriched. From Juarez et al. [9].

SNe can rapidly enrich the BLR to super-solar metallicities (more specifically, a SN rate of $10^{-4} \mathrm{yr}^{-1}$ can enrich the BLR to $\mathrm{Z}>\mathrm{Z}_{\odot}$ in less than $10^{8}$ years). More puzzling is that such huge metallicities are not found in the stellar population of massive galaxies in the local universe. This implies that the high metallicity BLR gas must be either expelled out of the galaxy, or mixed with lower metallicity gas in the host galaxy before forming stars.

\section{The iron and carbon problems}

Iron is mostly produced by SNIa, most of which are expected to explode on timescales longer than $\sim 1$ Gyr. Hence, the abundance of iron relative to $\alpha$-elements (e.g. $\mathrm{O}, \mathrm{Ne}$, 
$\mathrm{Mg}$, which are promptly produced by SNII on very short timescales) is regarded as a "clock" of star formation. The lack of evolution of the the FeII/MgII ratio as a function of redshift, suggests a lack of $\mathrm{Fe} / \alpha$ evolution out to $\mathrm{z}=6.4$. This result is puzzling, since at $z>5$ the age of the universe is less than $1 \mathrm{Gyr}$, hence stellar evolution should fall short of time to produce SNIa. Some possible scenarios can explain this paradox. First, the UV iron bump may not be a good tracer of iron abundance at all. Indeed the UV Fe bump is a strong coolant of the BLR and, therefore, its intensity may remain constant independently of the iron abundance. A better tracer of the iron abundance is probably the optical Fe bump at $\lambda \sim 4500[10,40]$, but which cannot be measured from ground at $\mathrm{z}>4$, so this test must await JWST. Another possibility is that the assumed iron enrichment timescale is wrong. Mannucci et al. [41, 42] show evidence for a significant population of SNIa, that explode on timescales as short as $10^{8} \mathrm{yr}$. However, Matteucci et al. [43] shows that these "prompt SNIa" represent only $~ 30 \%$ of the total population integrated over long time scales, hence this scenario may not provide a viable solution.

A similar timescale problem applies to carbon. Carbon is mostly produced by AGB stars and planetary nebulae. Although the first AGBs are produced as soon as $50 \mathrm{Myr}$ after the onset of star formation, most of the carbon production occurs after $\sim 1 \mathrm{Gyr}$, resulting into a delayed carbon enrichment relative to $\alpha$-elements. The finding that the ratio (SiIV+OIV])/CIV does not evolve with redshift, out to $\mathrm{z}=6.4$, suggests that the abundance of carbon relative to the $\alpha$-elements does not evolve, contrary to the expectation that the carbon abundance should drop at $\mathrm{z}>5$ because stellar evolutionary timescales fall short of time to produce carbon. This "carbon problem" is not only found in the BLR, but it is probably an issue arising also from carbon tracers on larger scales in the host galaxy (carbon lines from the narrow line region, as well as [CII] and $\mathrm{CO}$ emission from the host galaxy). Both iron and carbon issues at $\mathrm{z} \sim 6$ remain open and certainly require additional observations and modelling to be tackled.

\section{Quasar metallicity and stellar mass}

The high metal abundances in quasars at high redshift (especially at $\mathrm{z} \sim 6$ ) require that the host galaxy has undergone a rapid and powerful star formation event. Infrared and $\mathrm{mm}$ observations have indeed revealed vigorous star formation in the host galaxies of distant quasars, although not ubiquitously [30, 31, 44, 45, 46]. However, a potential problem is the comparison of the high level of metal enrichment with the estimated galaxy mass. Indeed, there are indications that high-z quasars are characterized by a galaxy-to-black hole mass ratio at least one order of magnitude lower than observed in local galaxies [47, 25]. While the ISM has already reached supersolar metallicities, more than $90 \%$ of the final stellar mass has still to be formed (to reach the local $\mathrm{M}_{\mathrm{BH}} / \mathrm{M}_{\text {galaxy }}$ relation). If confirmed with additional observations on a larger sample of quasars, these results may be challenging to explain by models of early $\mathrm{BH}$-galaxy coevolution.

\section{REFERENCES}

1. Fan, X., et al. 2006, AJ, 131, 1203 
2. Willott, C. J., et al. 2007, AJ, 134, 2435

3. Hamann, F., \& Ferland, G. 1999, ARA\&A, 37, 487

4. Nagao, T., Marconi, A., \& Maiolino, R. 2006, A\&A, 447, 157

5. Warner, C., Hamann, F., \& Dietrich, M. 2004, ApJ, 608, 136

6. Shemmer, O., Netzer, H., Maiolino, R., Oliva, E., Croom, S., Corbett, E., \& di Fabrizio, L. 2004, ApJ, 614,547

7. Pentericci, L., et al. 2002, AJ, 123, 2151

8. Jiang, L., et al. 2007, AJ, 134, 1150

9. Juarez, Y., Maiolino, R., Mujica, R., Pedani, M., Marinoni, S., Nagao, T., Marconi, A., \& Oliva, E. 2009, A\&A, 494, L25

10. Verner, E., et al. 2004, ApJ, 611, 780

11. Freudling, W., Corbin, M. R., \& Korista, K. T. 2003, ApJ, 587, L67

12. Iwamuro, F., et al. 2004, ApJ, 614, 69

13. Maiolino, R., Juarez, Y., Mujica, R., Nagar, N. M., \& Oliva, E. 2003, ApJ, 596, L155

14. Kurk, J. D., et al. 2007, ApJ, 669, 32

15. Dietrich, M., Hamann, F., Appenzeller, I., \& Vestergaard, M. 2003, ApJ, 596, 817

16. Sameshima, H., et al. 2009, arXiv:0902.2057

17. De Breuck, C., Röttgering, H., Miley, G., van Breugel, W., \& Best, P. 2000, A\&A, 362, 519

18. Vernet, J., et al. 2001, A\&A, 366, 7

19. Iwamuro, F., et al. 2003, ApJ, 598, 178

20. Nagao, T., Maiolino, R., \& Marconi, A. 2006, A\&A, 447, 863

21. D'Odorico, V., et al. 2004, MNRAS, 351, 976

22. Gabel, J. R., Arav, N., \& Kim, T.-S. 2006, ApJ, 646, 742

23. Walter, F., et al. 2003, Natur, 424, 406

24. Carilli, C. L., et al. 2007, ApJ, 666, L9

25. Maiolino, R., et al. 2007, A\&A, 472, L33

26. Maiolino, R., et al. 2005, A\&A, 440, L51

27. Iono, D., et al. 2006, ApJ, 645, L97

28. Walter, F., Riechers, D., Cox, P., Neri, R., Carilli, C., Bertoldi, F., Weiss, A., \& Maiolino, R. 2009, Nat, 457, 699

29. Maiolino, R. 2008, New Astronomy Review, 52, 339

30. Beelen, A., et al. 2006, ApJ, 642, 694

31. Wang, R., et al. 2007, AJ, 134, 617

32. Maiolino, R., Schneider, R., Oliva, E., Bianchi, S., Ferrara, A., Mannucci, F., Pedani, M., \& Roca Sogorb, M. 2004, Natur, 431, 533

33. Stratta, G., Maiolino, R., Fiore, F., \& D'Elia, V. 2007, ApJ, 661, L9

34. Granato, G. L., et al. 2004, ApJ, 600, 580

35. Di Matteo, T., Springel, V., \& Hernquist, L. 2005, Nat, 433, 604

36. Hopkins, P. F., Hernquist, L., Cox, T. J., \& Kereš, D. 2008, ApJS, 175, 356

37. Menci, N., Fiore, F., Puccetti, S., \& Cavaliere, A. 2008, ApJ, 686, 219

38. Li, Y., et al. 2007, ApJ, 665, 187

39. Maiolino, R., et al. 2008, A\&A, 488, 463

40. Netzer, H., \& Trakhtenbrot, B. 2007, ApJ, 654, 754

41. Mannucci, F., Della Valle, M., Panagia, N., Cappellaro, E., Cresci, G., Maiolino, R., Petrosian, A., \& Turatto, M. 2005, A\&A, 433, 807

42. Mannucci, F., Della Valle, M., \& Panagia, N. 2006, MNRAS, 370, 773

43. Matteucci, F., et al. 2006, MNRAS, 372, 265

44. Lutz, D., et al. 2007, ApJ, 661, L25

45. Lutz, D., et al. 2008, ApJ, 684, 853

46. Maiolino, R., Shemmer, O., Imanishi, M., Netzer, H., Oliva, E., Lutz, D., \& Sturm, E. 2007, A\&A, 468, 979

47. Walter, F., et al. 2004, ApJ, 615, L17 